\title{
Transient Rocket-Engine Gas Flow in Soil
}

\author{
Ronald F. ScOtT* \\ California Institute of Technology, Pasadena, Calif. \\ AND \\ HoN-YIM Ko† \\ University of Colorado, Boulder, Colo.
}

\begin{abstract}
As a result of an examination of the behavior of soils subjected to rocket and jet exhaust gases, it was concluded that the gas pressure in the pores of the soil played an important part in the process of soil removal which occurred. Consequently, a numerical study of axially symmetric isothermal nonlinear gas flow in a porous medium was undertaken. The paper presents the results and interpretations of the calculations for the particular boundary pressure conditions developed by a Surveyor spacecraft vernier engine mounted $12 \mathrm{in}$. above a granular material.
\end{abstract}

\section{Introduction}

$\mathbf{A}^{\mathrm{P}}$ PROPOSED experiment for the Surveyor spacecraft sometime after a successful lunar landing is the firing of its vernier rocket engines. Since Surveyors I and III have demonstrated the existence of a granular medium at the moon's surface, the question of the prediction and interpretation of the effect of the firing into a soil under appropriate circumstances arises. In addition, the landing of the Apollo Lunar Module on the moon will require the continuous firing of the vehicle's engine to a distance of a few feet above the lunar surface. The consequences of this must be reliably estimated in advance, since the effects may require close consideration of the final stages of the LM trajectory. A preliminary Surveyor experiment will be of value in conducting LM studies.

During the first lunar day of Surveyor I's life, consideration was given to a test employing the vernier engines used in controlling the spacecraft's descent, although there was doubt both as to their actual operation and the thrust level they would achieve. At the time, it was, of course, already known that the lunar surface was soil-like, so that the hazard to the spacecraft of firing the verniers had to be assessed. There were two dangers. One consisted in the possibility that a thrust level might be reached which could overturn the spaceeraft. The second danger, which concerns this paper, was that a successful firing, which did not overturn the spacecraft, might however cover it with soil expelled from the lunar surface below the engine nozzles. This material would impair the efficiency of the thermal control surfaces and might also come in contact with the camera mirror and other sensitive surfaces.

A number of studies were therefore initiated to clarify the questions relating to a test firing of the vernier engines. One of these involved test firings of a Surveyor vernier engine in a rocket engine vacuum test chamber at the JPL-Northrop facility at Edwards Air Force Base, Calif., in June 1966 in order to simulate the Surveyor I temperature conditions at the lunar surface. The senior author suggested that some of these firings be carried out on a soil simulating the observed lunar surface material as well as it could be judged, at the level of the lunar surface below the Survevor I vernier engine nozzle, a distance of about $12 \mathrm{in}$. No attempt was made to

Received February 20, 1967; revision received July 28, 1967. The analytical work described in this paper was supported under California Institute of Technology, Jet Propulsion Laboratory, Engineering Contract 69811.

* Professor of Civil Engineering.

† Assistant Professor of Civil Engineering. simulate lunar gravity. The tests will not be described in detail. The depressions in the surface resulting from one test firing at $20-\mathrm{lb}$ thrust and $\frac{1}{2}$-sec duration are shown in Fig. 1 and were $\frac{1}{2}$ in. to $\frac{3}{4}$ in. deep. Partly as a result of the tests at Edwards, it was decided not to attempt a test firing of the Surveyor I verniers during the first lunar day.

To clarify the soil behavior during the firings, further tests were carried out in different soils at the JPL-Northrop facility in September 1966. A photograph of the soil surface after one test is shown in Fig. 2. In the fine-grained soil in the June 1966 tests, the area below the axis of the jet was not eroded, and in fact, material was deposited on it. This region was surrounded by an annular trough from which the soil had been removed. In the coarser soil of the September tests, a central crater (Fig. 2) was surrounded by a circular rim whose crest was at the original soil surface. Outside this rim was another annular trough or moat. High-speed motion pictures were made of the events during and on cessation of the firing. These movies and the soils behavior will be discussed in the next section.

\section{Previous Studies of Rocket Exhaust-Soil Interaction}

Roberts ${ }^{2}$ analyzed the interaction of a gas jet with a granular medium in a vacuum from the point of view of erosional removal of the particles at the surface for a jet whose axis is normal to the surface. He formulated equations describing the phenomenon in terms of the distribution of fluid shearing stresses at the surface of the medium and the fluid drag on the particles. The behavior of a granular material subjected to fluid flow at its surface has, of course, been extensively studied in order to understand both subaerial and subaqueous erosional processes. ${ }^{3.4}$ Experiments were earried out by Land and Clark $^{5}$ on uniformly-sized soils whose erosion was measured during the operation of the jet by means of an X-ray technique; the results compared with Roberts' theory. The behavior of soils containing a wide distribution of sizes has apparently not been studied. The effect of the flow of the jet gas into and through the pores of the soil was not examined, and the changing geometry of the surface during erosion, with its interaction with the jet behavior, was not taken into account in Roberts' analysis. In the formulation of the equations describing the gas flow, Roberts ${ }^{2}$ assumed that the surface of impingement was impermeable. The nature of the flow near a rigid but permeable surface would be different because of the flow in the porous medium, but this problem does not seem to have been examined, to the authors' knowledge. 
In their experimental investigation of the impingement of a jet on a granular surface, Land and Clark ${ }^{5}$ observed slumping of the crater following shutdown of the jet. In the finer grained materials this slumping was sufficient to destroy entirely the crater formed during the firing. They attributed this effect to discharge of jet gases from the soil to the ambient vacuum when the jet was stopped. The effect of pore gases on the penetration of projectiles into soils in air and vacuum was observed by Roddy et al. ${ }^{6}$

In the high-speed motion pictures made during the September 1966 tests at Edwards Air Force Base, a distinct disturbance of the soil surface took place immediately under the engine nozzle as soon as firing stopped. In Fig. 3a is shown one of the movie frames during firing about 50 milliseconds from engine shutdown. This soil configuration remained relatively unchanged during the last one or two tenths of a second of the process. The soil appearance a few milliseconds after shutdown is shown in Fig. 3b, in which an upwelling of the central region of soil can be seen.

As a result of these and other observations, it was concluded that gas flow through the pores of the soil under some soil conditions contributes significantly to the phenomenon of soil removal by a rocket engine or jet directed at the soil surface. In particular, the appearance of a crater surrounded by a rim beyond which a further annular crater or moat had developed, as shown in Fig. 2, does not seem to be predictable by the mechanism of erosion by entrainment as developed by Roberts.

Consequently a study was undertaken of gas flow through a porous medium under conditions directly applicable to the firing of the Surveyor spacecraft's vernier engines at the lunar surface. The analysis and its results are described in the following sections.

\section{Formulation of the Problem}

The isothermal transient flow of an ideal gas through a porous medium when the flow obeys Darcy's Law is described by the following second-order, nonlinear equation ${ }^{7}$ :

$$
\nabla^{2} p^{2}=(2 n \mu / k)(\partial p / \partial t)
$$

where $p=$ pressure, $n=$ porosity of the porous medium, $\mu=$ viscosity of gas in the medium, and $k=$ permeability of the medium to gas flow.

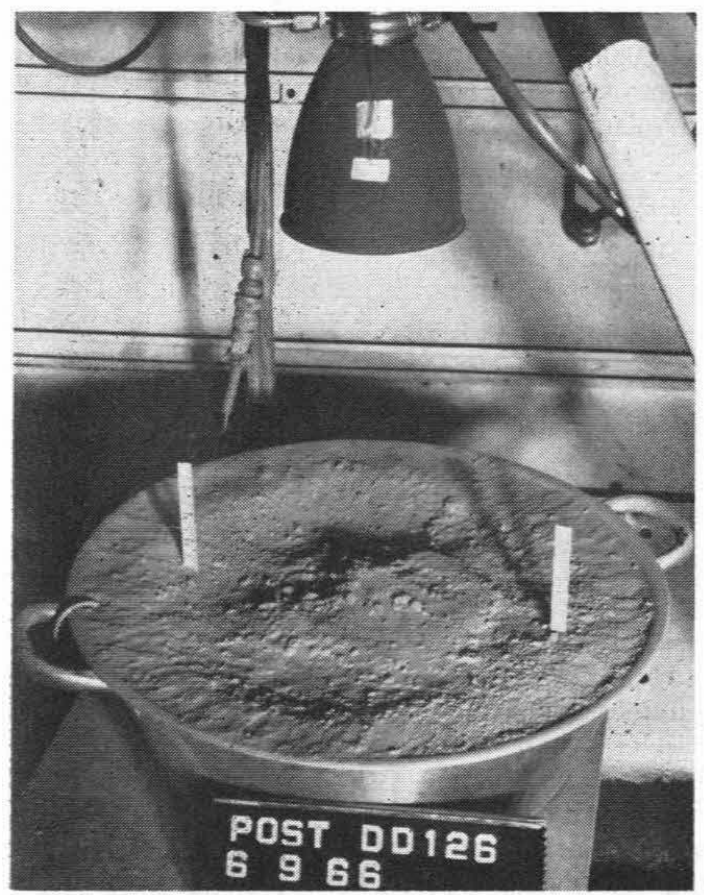

Fig. 1 Soil surface after test, June 1966.

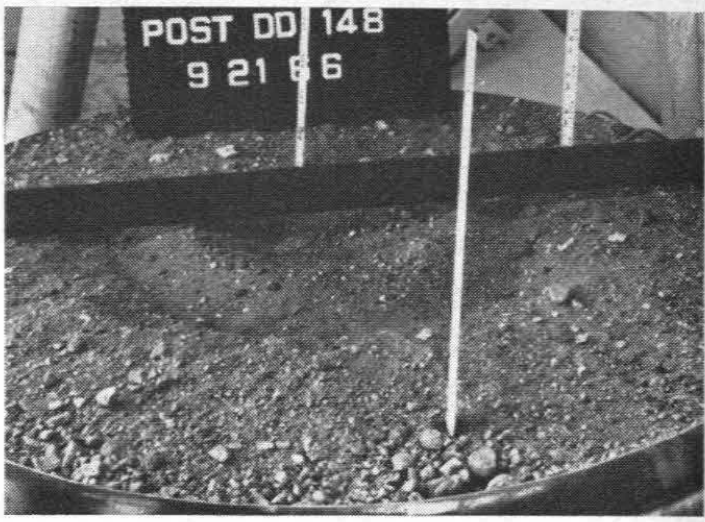

Fig. 2 Soil surface after test, Sept. 1966.

Exact solutions to this equation are, in general, difficult to obtain. An approximate solution has been obtained to the one-dimensional transient flow problem $^{8}$ and numerical methods ${ }^{9}$ as well as electrical analogs ${ }^{10}$ have been used to study the one-dimensional problem. A three-dimensional problem with radial symmetry has also been studied. ${ }^{11}$ The two-dimensional steady-state flow of air through show was treated analytically by Yen and Fisher. ${ }^{12}$

In the following, the transient flow of exhaust gases from a rocket engine firing onto a granular soil under vacuum conditions is studied numerically. The boundary conditions prevailing during the firing test are simulated in the calculations.

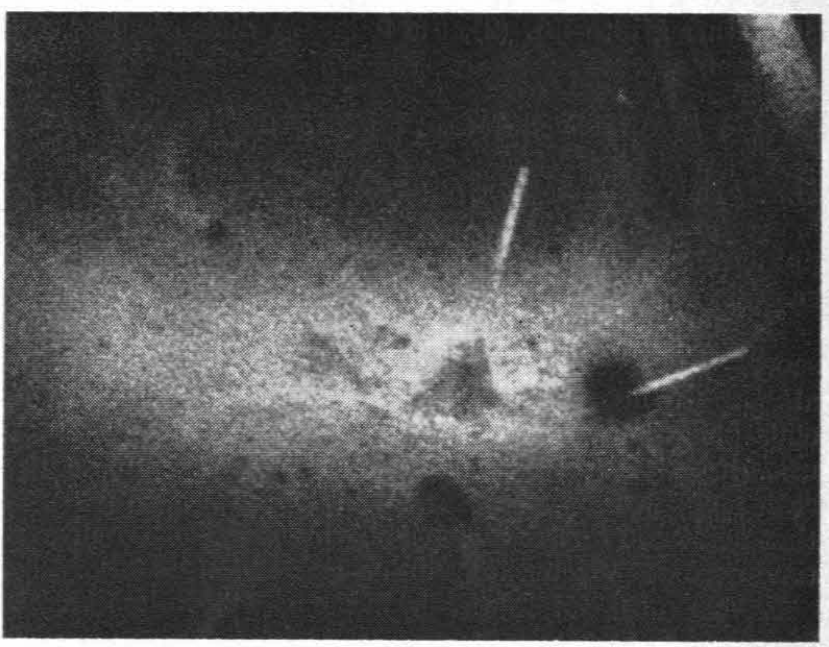

Fig. 3a Soil surface during firing, Sept. 1966.

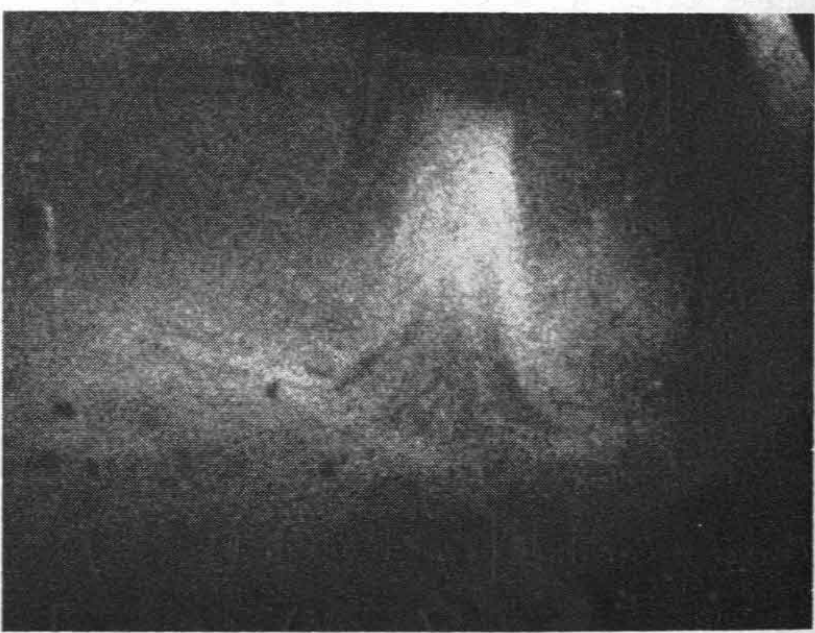

Fig. 3b Soil surface immediately after shutoff, Sept. 1966. 


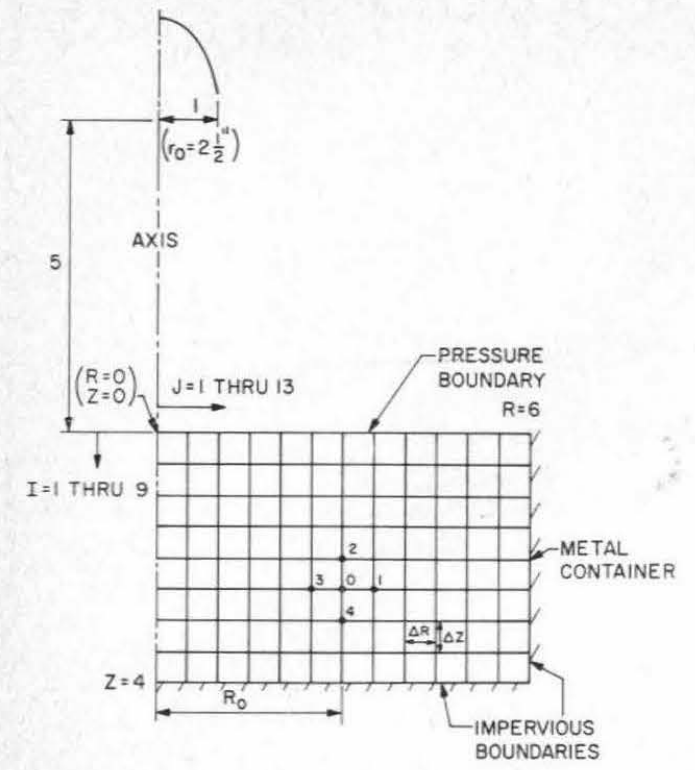

Fig. 4 Radial network, mesh size and boundary conditions.

Equation (1) can be written, in the case of radial symmetry, as

$$
\frac{\partial^{2} p^{2}}{\partial z^{2}}+\frac{1}{r} \frac{\partial}{\partial r}\left(r \frac{\partial p^{2}}{\partial r}\right)=\frac{2 n \mu}{k} \frac{\partial p}{\partial t}
$$

Let $P=p / p_{0}, R=r / r_{0}, Z=z / r_{0}$, and $T=\left(k p_{0} / 2 \mu n r_{0}^{2}\right) t$. Substituting into Eq. (2), the following dimensionless equation is obtained:

$$
\partial^{2} P^{2} / \partial Z^{2}+(1 / R)(\partial / \partial R)\left[R\left(\partial P^{2} / \partial R\right)\right]=\partial P / \partial T
$$

Here $p_{0}$ is a characteristic pressure, actually taken to be 1 psi; $r_{0}$ is the radius of the jet nozzle. It can be seen that the soil properties $k$ and $n$ only enter into the equation in the time scale, the characteristic time being equal to $2 \mu n r_{0} / k p_{0}$.

Equation (3) can be written in finite difference form in several ways. One is the implicit form, as used by Bruce et al. ${ }^{10}$ which involves solving a set of simultaneous algebraic equations for each time step. Another is the explicit form using a progressive type of solution in which the pressure at each point in the medium is calculated from the pressure at that point and those at its neighboring points at the previous time step. The latter procedure is followed in this study.

Equation (3) can be written in the explicit finite difference form $^{12}$ as

$$
\begin{aligned}
& \frac{P_{0 . T+\Delta T}-P_{0, T}}{\Delta T}=\frac{1}{(\Delta Z)^{2}}\left[P_{2, T^{2}}+P_{4, T^{2}}-2 P_{0, T^{2}}\right]+ \\
& \frac{1}{(\Delta R)^{2}}\left[\left(1+\frac{\Delta R}{2 R_{0}}\right) P_{1, T^{2}}+\left(1-\frac{\Delta R}{2 R_{0}}\right) P_{3, T^{2}}-2 P_{0, T^{2}}\right]
\end{aligned}
$$

where the notation follows that of Fig. 4. $\quad P_{0, T}$ is the dimensionless pressure at point 0 with coordinates $\left(Z_{0}, R_{0}\right)$ at the time $t ; P_{2, T}$ is the dimensionless pressure at point 2 at time $t$, where point 2 has coordinates $\left(Z_{0}-\Delta Z, R_{0}\right)$ and so on. The sizes of the mesh in the $R$ and $Z$ directions are $\Delta R$ and $\Delta Z$, respectively.

If $\Delta R=\Delta Z$, i.e., for a square mesh, then, letting $M=$ $\Delta T /(\Delta Z)^{2}$, Eq. (4) becomes

$$
\begin{array}{r}
P_{0, T+\Delta T}=P_{0, T}+M\left[P_{2, T^{2}}+P_{4, T^{2}}+\left(1+\frac{\Delta R}{2 R_{0}}\right) \times\right. \\
\left.P_{1, T^{2}}+\left(1-\frac{\Delta R}{2 R_{0}}\right) P_{3, T^{2}}-4 P_{0, T^{2}}\right]
\end{array}
$$

It can, therefore, be seen that the pressure at any point in the medium at any particular time can be calculated by means of Eq. (5) from the pressures at that point and its neighboring points at a time $\Delta T$ before. If the initial pressure distribution is known, a stepwise determination of the pressure distribution can be obtained by repeated use of Eq. (5) at each point.

In Eq. (5) a singularity appears at points on the axis where $R_{0}=0$. For those points, a different equation has to be derived by considering that there is no flow of gas across this axis of symmetry. The equation is

$$
P_{0, T+\Delta T}=P_{0, T}+M\left[P_{2, T^{2}}+P_{4, T^{2}}+4 P_{1, T^{2}}-6 P_{0, T^{2}}\right]
$$

\section{Calculational Stability Considerations}

Only two of the three quantities, $\Delta T, \Delta Z$, and $M$ can be chosen independently. The smaller the values chosen for $\Delta T$ and $\Delta Z$, the better an approximation the difference equation (5) is to the differential equation (3). As $\Delta Z$ decreases, the number of grid points required to cover a specific area increases as $1 /(\Delta Z)^{2}$. Therefore, for practical reasons, $\Delta Z$ cannot be too small.

The value of $M$ to be chosen has to depend on the stability of the problem. Instability in the solution develops due to the accumulation of round-off errors in the numerical calculations and to truncation errors introduced by the omission of higherorder terms when the difference equations were written. For the linear, one-dimensional diffusion equation

$$
\partial^{2} P / \partial Z^{2}=\partial P / \partial T
$$

it has been shown ${ }^{12}$ that for $M>\frac{1}{2}$, a diverging solution is obtained, whereas for $M=\frac{1}{2}$, an oscillating solution results. It can be shown that in the linear, two-dimensional case, the requirement for stability is $M \leq \frac{1}{4}$. In the nonlinear, onedimensional case, Bruce et al. ${ }^{10}$ showed that for $M=\frac{1}{2}$, an unstable solution was obtained; for $M=1 / 3.64$ an oscillating solution was obtained; and for $M=\frac{1}{8}$ a stable solution was obtained. In the present nonlinear, three-dimensional problem with radial symmetry, a eomplication arises due to the presence of the radial terms $\Delta R / 2 R_{0}$, resulting in changing values of $M$ as $R_{0}$ changes. A trial-and-error procedure was adopted here by solving the problem with different values of $M$ and examining the stability of the results. It was found that for $M=0.1$, an unstable solution is obtained, whereas for $M=0.05$, a stable one resulted. Therefore, a value of $M$ equal to 0.05 was used in the calculations. The solutions at long times were also checked by comparison with the steadystate Laplacian in the pressure squared.

\section{Solution of the Problem}

To simulate the actual firing conditions in the Edwards tests, as shown in Fig. 4, the geometric configuration of Fig. 4 was chosen for the granular medium. The size of mesh chosen was $\Delta Z=\Delta R=\frac{1}{2}$, and the time step $\Delta T=M(\Delta Z)^{2}$ $=0.0125$. An initial pressure of 0.02 psi is assumed to exist in the pore fluid of the soil and at the surface, and for time $T \geq 0$, the surface of the soil is assumed to be subjected to a static pressure exerted by the exhaust gas from the jet. A normal surface pressure distribution, centered on $R=0$, is assumed, with the standard deviation of the normal curve equal to the nozzle radius; that is, for $R>3$ on the surface, the pressure remains constant at $0.02 \mathrm{psi}$. The normalizing pressure $p_{0}$ is chosen to be $1 \mathrm{psi}$ and the dimensionless pressure $P$ is numerically equal to the pressure $p$. A peak surface pressure of 2 psi is used.

The sides and bottom of the soil container are impervious to gas flow. To represent these impervious boundaries in the actual calculations an imaginary point is assumed to exist beyond the boundary at a distance of one mesh size away. This point is given a pressure equal to that at the correspond- 
ing point in front of the boundary (its mirror image). For example, for a calculation at a point 0 coinciding with the bottom of container, Eq. (5) can be used with the provision that $P_{4, \tau}$ is taken equal to $P_{2, T}$.

It is possible to examine the case of time-dependent surface pressure distribution due to, say, the gradual approach of the jet to the surface, but in the present study a constant pressure is used to simulate the firing of the Surveyor vernier engine at a constant height. It is important to note that during the firing of the jet, erosion of the soil surface by the exhaust gas flowing along the surface generally occurs. However, in this study, a surface configuration that does not change with time is assumed. This point will be discussed in more detail in a later section.

The shutdown of the engine can be simulated by removing the imposed pressure distribution on the surface. The engine can be fired for different periods of time and then shut off. The equations governing the diffusion of gas out of the soil after engine shutdown are exactly the same as those during firing. Only a change in the surface boundary conditions is required. The diffusion of gas through the medium during firing is studied as well as the diffusion subsequent to shutdown after firing for different times, and the results are presented in the following section.

\section{Results of Pressure Calculation}

The pressure distributions of the gas in the soil at different times after the engine is turned on are shown in Fig. 5. The pressures at different depths below the center of the engine are shown as functions of time in Fig. 6 and the pressures at different radial distances from the center at the bottom of the first layer are shown in Fig. 7. It can be seen that at $T=5$, the pressures at most of the points near the surface have reached steady values. Hence, it can be assumed that steady state of flow is reached at about $T=5$. Equation (5) can be used to check whether the steady state has been reached or not. Under the steady state, the parenthesis on the righthand side should vanish at all points in the medium. It is, indeed, found that this condition is true for all the points except those near to the bottom boundary and those near to the comer where the two impermeable boundaries meet.
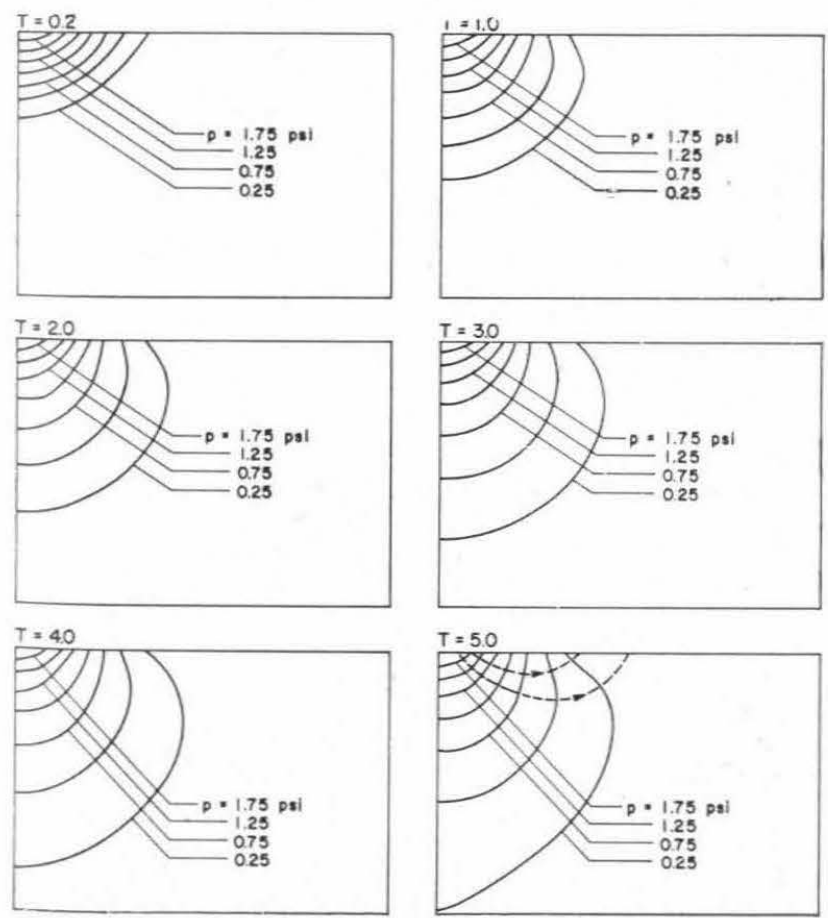

Fig. 5 Pressure distributions at different times during firing.

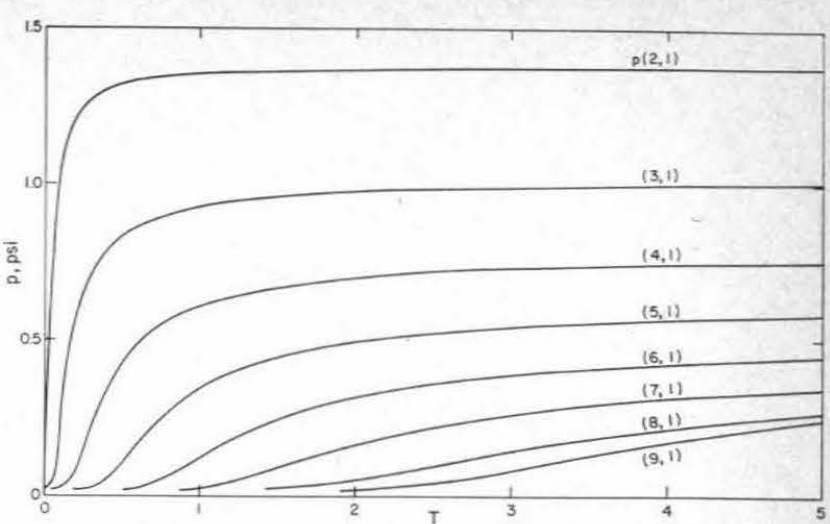

Fig. 6 Pressure vs time at varying depths under the center of the jet.

For firing up to a certain time, say, $\frac{1}{2}$ sec, whether the steady state has been reached or not depends on the characteristics of the soil, since the soil property is represented in the group of terms defining the characteristic time. Under the circumstances of firing, the gas viscosity is about $1 \times 10^{-6} \mathrm{lb}$ sec $\mathrm{ft}^{-2}$ and the soil's permeability, varying with grain size, has values of $10^{-9}-10^{-10} \mathrm{ft}^{2}$ for a coarse sand (grain size 1000 $500 \mu$ ) to $10^{-18}-10^{-14} \mathrm{ft}^{2}$ for a fine silt (grain size $50-10 \mu$ ). Consequently, the characteristic time can vary from $10^{-1} \mathrm{sec}$ in a coarse sandy soil to $10^{4}$ secs in a fine granular medium, for the Surveyor mode and pressure in question.

For a coarse-grained, very permeable soil under the present conditions, it seems likely that firing for $\frac{1}{2}$ sec may develop the steady-state flow condition whereas in a fine-grained very impermeable soil, the steady state may take minutes to develop in the test under discussion. In order to study the shutdown phenomenon in different soils, it is therefore necessary to turn off the engine at different times, after the steady state has been reached, as well as before.

Three cases have been studied. In the first, the engine is shut off at $T=5$ and calculations are continued up to $T=6$. In the second and third cases the engine is shut off at $T=2$ and $T=0.2$, respectively, and the calculations are continued up to $T=5$ and $T=2.2$, respectively. The pressure distributions in these three cases are shown at different times after shutdown in Fig. 8.

\section{Considerations Pertaining to Surface Erosion}

\section{During Firing}

From the pressure distribution obtained in the previous section, the direction of gas flow at a point can be obtained since, in the isotropic medium, it takes place along the direc-

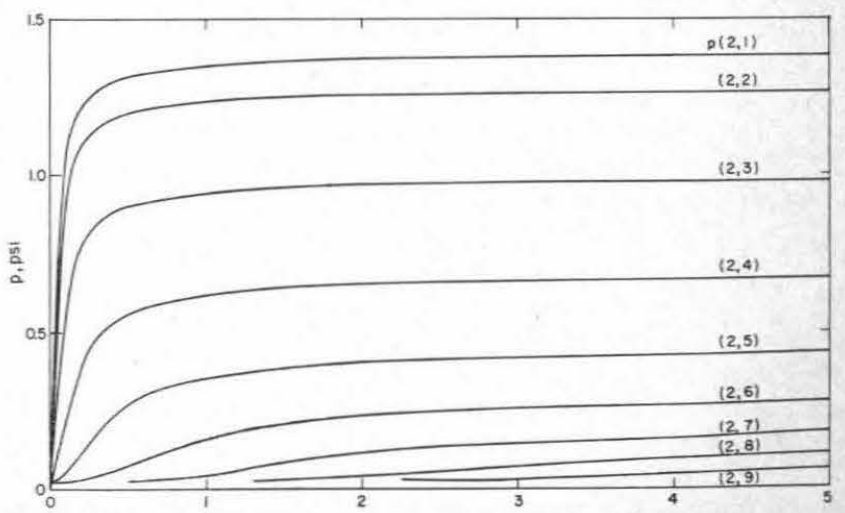

Fig. 7 Pressure at $Z=\frac{1}{2}$ vs time at varying radial distances. 


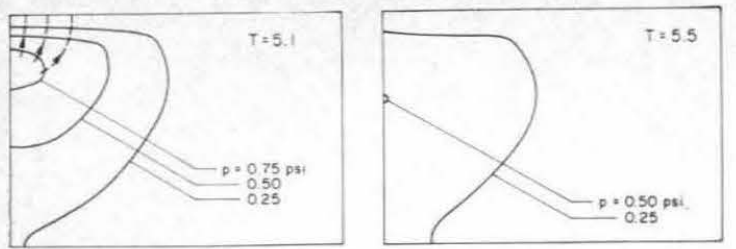

AFTER SHUT - OFF AT T $=5.0$

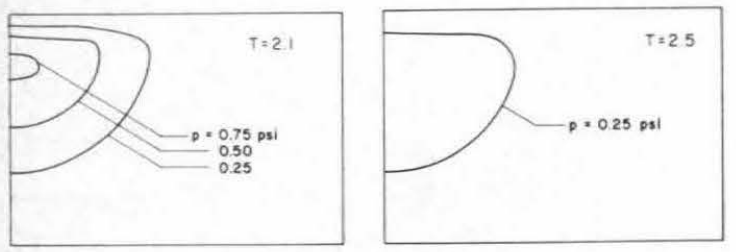

AFTER SHUT - OFF AT $T=2.0$
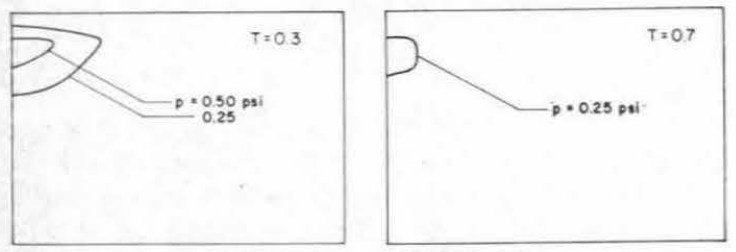

AFTER SHUT - OFF AT $T=0.2$

Fig. 8 Pressure distributions at different times after shutoff.

tion of steepest pressure gradient. The body forces on an element of soil due to the flow of gas through it will be proportional to the pressure gradient and act in the same direction as the flow. The general direction of the flow during firing is downwards into the soil directly underneath the jet and upwards out of the soil in surface regions three or four characteristic lengths from the center, as shown in Fig. 5. In the latter regions, the rate of upward flow, or the pressure gradient, in a real test increases as firing goes on until such a time that the vertical upward component of the force exerted by the flow exceeds the weight of the soil. The region of soil where this condition is reached becomes potentially unstable. Whether or not this part of the soil will be lifted up and removed by the gas stream along the surface depends on the strength of the soil. In particular, since the net vertical force has become zero (the effective stress, as used in soil mechanics, has become zero), the part of the soil strength which depends on the stresses acting between grains (effective stresses) is zero, and therefore only the cohesion of the soil is important in holding the soil down. If the soil is completely cohesionless, then the entire region of soil in which the force due to the upward flow of gas exceeds the weight of the soil may be removed. However, in practice, once erosion begins to take place, the geometrical configuration of the surface and consequently of the flow region changes and solutions previously obtained will no longer be valid for subsequent times. It is, therefore, to be remembered that the calculations of pressures described previously assume that the surface boundary and the flow properties of the region do not change with time. This assumption is then equivalent to considering that the soil grains in the potentially unstable regions are supported by the gas flow (the material has been liquefied or become quick) without being carried away by the exiting gas.

\section{After Shutdown}

The same kind of instability phenomenon occurs after the shutdown of the engine. The gas flow directions are indicated in Fig. 8. It can be seen from Fig. 8 that very steep pressure gradients appear near the center of the region after the shutdown. All the calculations subsequent to shutdown are valid only if it is assumed, as before, that this central region stays in place.

\section{Erosion Estimate}

It is interesting to make an estimate of the extent of the potentially unstable region both during firing and after shutdown. Since the weight of the soil enters into this consideration, the same soil will exhibit different erosion amounts under earth and lunar gravity conditions. The effect of gravity is considered in the following calculations.

A square element, whose corners are the grid points of the mesh used in the pressure calculations, is assumed to be subjected to uniform pressures acting on its four sides, which are the average of pressures at the corresponding pair of grid points at the corners. In addition, the weight of the element is assumed to act on the bottom of the element. (The unit weight of the soil is taken to be $100 \mathrm{lb} / \mathrm{ft}^{3}$ on earth and 16.7 $\mathrm{lb} / \mathrm{ft}^{3}$ on the moon.) The resultant of the vertical forces acting on the upper and lower faces of the element and the weight of the element is calculated for each element. If the net force for an element on the surface acts upwards, then the weight of this element is neglected in calculations for the element below it. This is equivalent to assuming that the top element is in a floating position and yet the flow boundary has not changed so significantly as to invalidate the solution of the finite difference equations for the flow problem. Such calculations are performed for each element during firing and after shutdown at different times under earth and then lunar gravity. From these calculations it is possible to define potentially unstable regions within which the soil would have been blown away if it possessed no cohesion. In Figs. 9-11, these potentially unstable regions are shown under different firing and shutdown conditions and comparisons are made for the effect of gravity.

It must be pointed out, however, that due to the discrete nature of the formulation of the problem, the first instant of instability (e.g., $T=1.0$ at $g$ in Fig. 9) may not be too meaningful. It is possible that instability occurs earlier than $T=1.0$. Also, for the same reason, the lines defining these
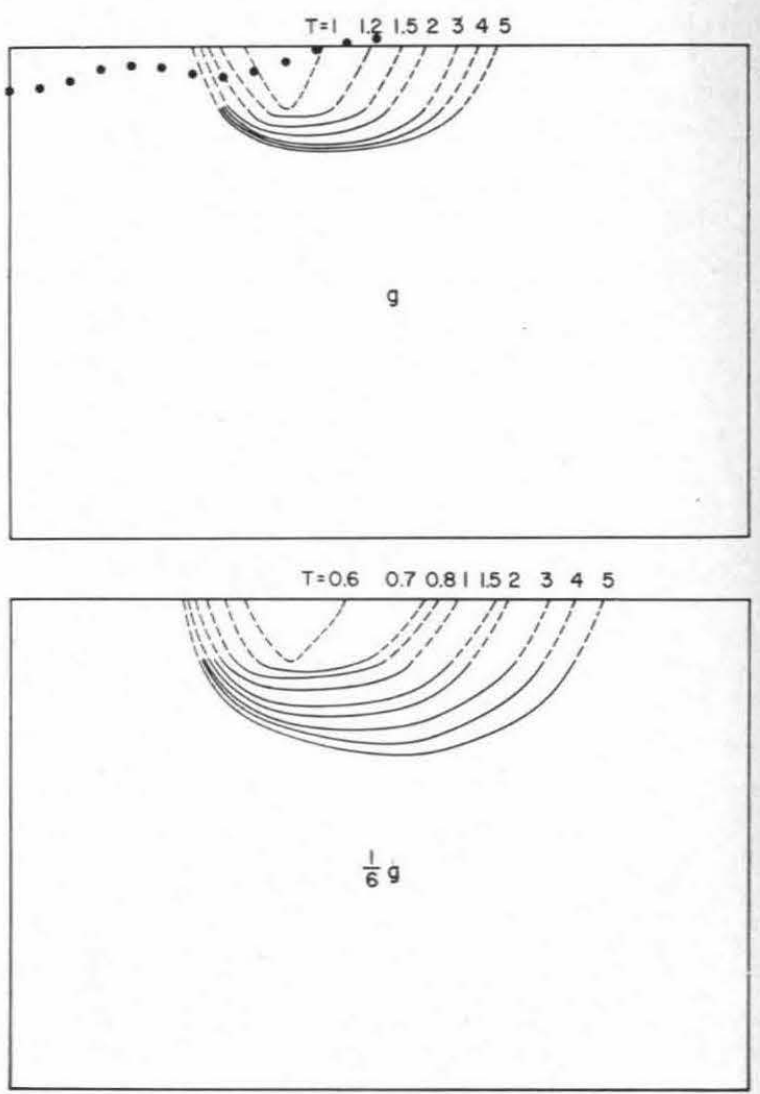

Fig. 9 Regions of potentially unstable soil at different times during firing to $T=5.0$, at $g$ and $\frac{1}{6} g$. 

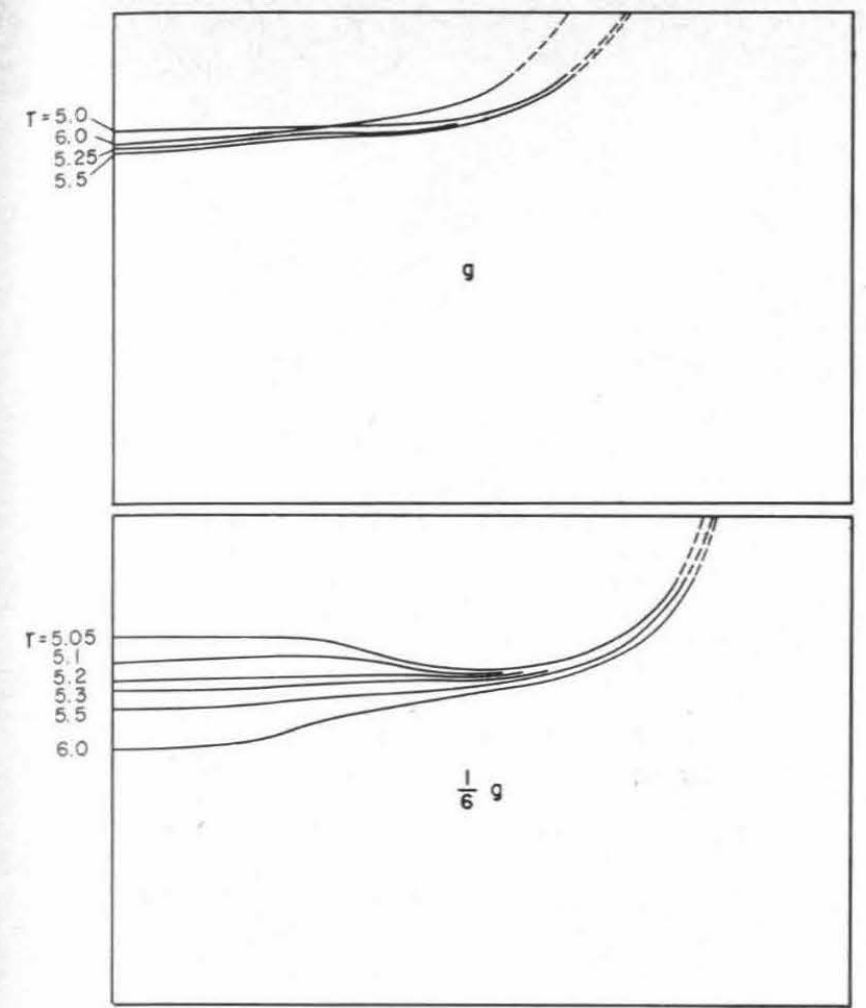

Fig. 10 Regions of potentially unstable soil at different times after shutoff at $\boldsymbol{T}=\mathbf{5 . 0}$, at $g$ and $\frac{1}{6} \mathrm{~g}$.

regions have to be extrapolated to the surface, because the resultant vertical forces on the first layer of elements are assumed to act at the bottom of the layer. However, the general pattern of the possible erosion is still valid. The finite difference grid can be made smaller if a more detailed study of soil removal near the surface is ever required.

In order to study the effect of the bottom boundary on the erosion pattern, a solution was obtained where the bottom impermeable boundary was located at a depth twice as great as in the previous solutions. The solution shows that the pressure distribution in the top half of the new region is almost identical to that in the region previously studied, except in the bottom two layers of the soil where the effect of an impervious boundary is most significant. The pressures near the surface, and hence the regions of potentially unstable soil, remain unaffected by moving the bottom boundary away from the surface. Therefore, the solutions obtained and the conclusions reached in this study are expected to be valid in situations where the engine fires onto a semi-infinite porous medium.

As stated earlier, only the cohesion of the soil, but not its frictional resistance, is effective in resisting erosion by the existing gas. To evaluate the effect of cohesion of the soil on the erosion pattern caused by the engine exhaust, a strength analysis can be performed from which it is possible to estimate the minimum cohesive strength of the soil required to prevent any soil removal down to a given depth.

\section{Conclusions}

It is suggested that the three processes, 1) erosion by entrainment of soil particles in the gas flow, 2) rapid cratering as a result of the jet-caused, normal surface gas pressure's exceeding the bearing capacity of the soil ${ }^{13}$ and 3 ) soil movement as a result of the upwards flow of gas through the pores of the soil during and at the end of firing, all occur as a result of the interaction of a jet with a granular medium. Which process predominates depends on the thrust level, height, and degree of expansion of the jet, and the length of firing in relation to

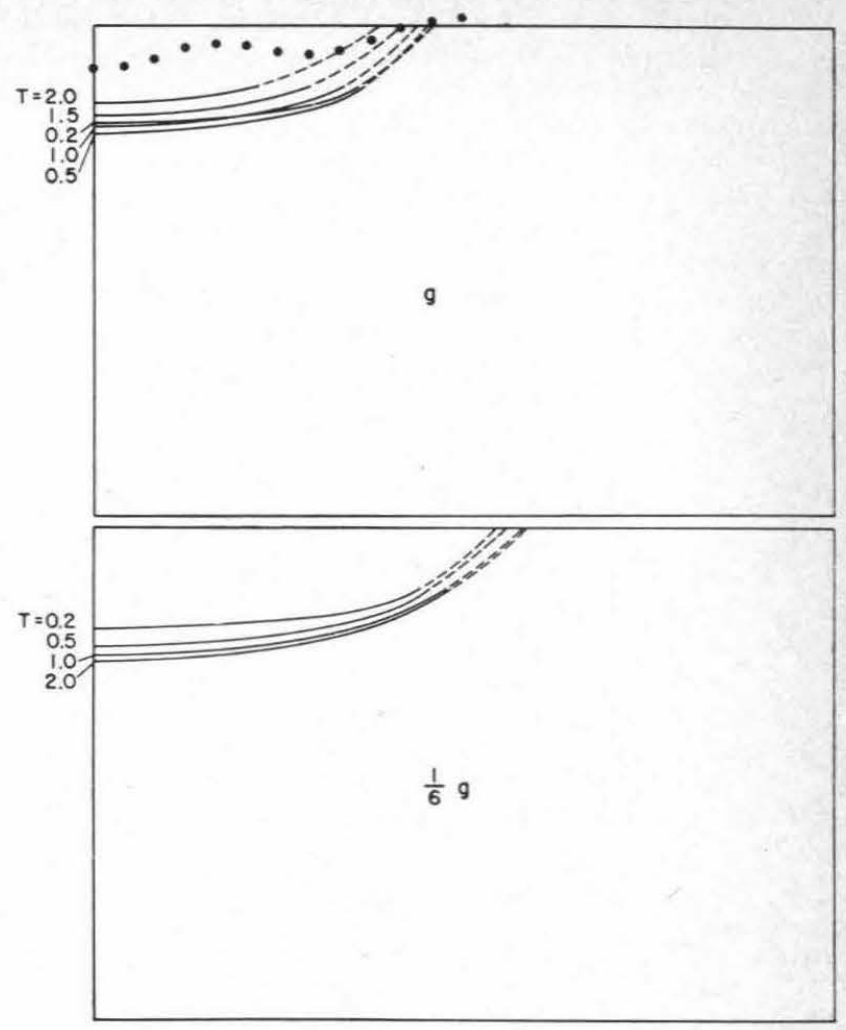

Fig. 11 Regions of potentially unstable soil at different times after shutoff at $T=0.2$, at $g$ and $\frac{1}{6} g$.

the soil characteristics of cohesion, grain size, and size distribution as they play a part in the entrainment processes and control the permeability. For high-static gas pressures at the ground surface, yielding that results in sudden or explosive cratering may occur. ${ }^{18}$ Pressures less than those required to cause such sudden failures cause both surface entrainment and gas flow into and through the granular medium. If the time of firing is short relative to the characteristic time of the system, little penetration of the gas pressures into the soil will occur, and the principal mechanism of soil removal will be particle entrainment by the lateral gas stream at the soil's surface.

However, for firing times long compared to the characteristic time, the flow through the soil can develop more fully, and it ean be seen from Fig. 8, for example, that an upward component of flow occurs in an annular region at the surface. This annulus does not, in general, correspond with the region of maximum surface shearing stresses caused by the jet, and it follows that, under the appropriate conditions, more than one region of soil removal can occur, as demonstrated, for example, in Fig. 2.

For all but very short firing times, cessation of firing with the subsequent reimposition of a uniform pressure smaller than the firing pressures at the soil surface, permits the gas stored in the soil to vent through the soil to the surface. With the highest gas pressures developed immediately under the jet this results in a circular region of uplifted soil in this position. This soil in most cases rises as the gas escapes and falls back to the surface again, usually in a loosened condition. The extent to which material is lost from the area depends on the dimensionless time duration of firing, the soil characteristics, and the gravitational field. This process is shown in Fig. 3b.

Figures 9-11 show separately the regions of potentially unstable soil developed during firing, and immediately following shutdown, respectively. In a real test, the effects are, of course, superimposed, and it will be seen that, depending on the characteristic time for the soil, various patterns of surface material removal can be obtained. For comparison with 
the computed results, the soil profile remaining at the end of the test shown in Fig. 2 has been plotted on Figs. 9 and 11. It would appear in this case that the profile best fits the computed results for shutdown at about the dimensionless time 0.1 , which gives a characteristic time for the soil of about 5 sec. It must be remembered that some of the soil falls back to the surface as the gas flow out of the soil diminishes.

In a cohesionless soil, the surface material will begin to blow away as soon as firing begins, and removal will continue during firing and at shutdown. However, a soil with cohesion will not erode on ignition of the rocket, since, as pointed out before, the cohesion prevents the removal of soil in the potentially unstable regions.

As firing continues, the net upward force on the unstable region in a given soil increases. If, at a subsequent stage, this gas-developed force exceeds the resisting force due to gravity and soil cohesion, the region will be ejected from the soil surface and removed. Consequently, in a soil with a small amount of cohesion, firing can take place for some time with little or no visible effect on the soil until chunks of the soil become detached at a time when the cohesive resistance of the soil is exceeded. As soil is usually inhomogeneous, these pieces of soil will not be removed uniformly and symmetrically around the impingement axis.

\section{References}

${ }^{1}$ Jaffe, L. D. et al., "Surveyor I: Preliminary Results," Science, Vol. 152, June 1966, pp. 1737-1750.

${ }^{2}$ Roberts, L., "The Action of a Hypersonic Jet on a Dusty
Surface," Paper 63--50, 1963, Institute of Aerospace Science. 3 Bagnold, R. A., The Physics of Blown Sand, W. Morrow, New York, 1943.

"Vanoni, V. A., "Sediment Transportation Mechanics: Initiation of Motion, Progress Report, Committee on Sedimentation," Proceedings of the American Society of Civil Engineers, Journal of the Hydraulics Division, Vol. 92, 1966, pp. 291-314.

${ }^{5}$ Land, N. S. and Clark, L. V., "Experimental Investigation of Jet Impingement on Surfaces of Fine Particles in a Vacuum Environment," TND-2633, Feb. 1965, NASA.

${ }^{6}$ Roddy, D. J., Rittenhouse, J. B., and Scott, R. F., "Dynamic Penetration Studies in Crushed Rock," AIAA Journal, Vol. 1, No. 4, April 1963, p. 868.

${ }^{7}$ Longwell, P. A., Mechanics of Fluid Flow, McGraw-Hill, New York, 1966.

${ }^{8}$ Aronofsky, J. S. and Jenkins, R., "Unsteady Flow of Gas through Porous Media, One-Dimensional Case," Proceedings of the First U.S. Congress of Applied Mechanics, 1950, p. 763.

${ }^{9}$ Green, L. and Wilts, C. H., "Nonsteady Flow of Gas through a Porous Wall," Proceedings of the First U.S. Congress of Applied Mechanics, 1950 , p. 777.

${ }^{10}$ Bruce, G. H., Peaceman, D. W., Rachford, H. H., and Rice, J. D., "Calculations of Unsteady-State. Gas Flow through Porous Media," Transactions of the American Society of Mining, Metallurgical, and Petroleum Engineers, Vol. 198, 1953, p. 79.

${ }^{11}$ Yen, Y.-C., and D. Fisher, "Isothermal Flow of Air in a Porous Medium into a Rectangular Sink," Journal of Geophysical Research, Vol. 69, 1964, p. 4211.

${ }^{12}$ Scott, R. F., Principles of Soil Mechanics, Addison-Wesley, Reading, Mass., 1963.

${ }^{13}$ Alexander, J. D., Roberds, W. M., and Scott, R. F., "Soil Erosion by Landing Rockets," Final Rept. Contract NAS9-4825, July 1966 , NASA. 